\title{
Clinical Outcomes of the Lateral Medullary Syndrome: A Case Report
}

\author{
Sakine Mazaherpur ${ }^{1}$, Avid Rokni ${ }^{1}$, Ehsan Bastani Far $^{1}$ and Alireza Abdi ${ }^{2,{ }^{*}}$ \\ ${ }^{1}$ Treatment Management of Social Security Organization of Khuzestan Province, Mahshahr, Iran \\ ${ }^{2}$ Nursing and Midwifery School, Kermanshah University of Medical Sciences, Kermanshah, Iran \\ "Corresponding author: Assistant Professor of Nursing, Nursing and Midwifery School, Kermanshah University of Medical Sciences, Kermanshah, Iran. Tel: +98-8338282102, \\ Email:a_abdi61@yahoo.com
}

Received 2017 October 20; Revised 2018 January 29; Accepted 2018 February 24.

\begin{abstract}
Introduction: Lateral medullary syndrome (LMS) is a rare stroke and often results from thrombosis or emboli of the vertebral artery or posterior-inferior cerebellar artery. There is a wide range of clinical manifestations found in this syndrome and treatment is done usually as symptomatic.

Case Presentation: We present the case of an Iranian 45-year-old man with a history of vertigo, ataxia, hiccup, and eye abnormalities lasting for three days in Mahshahr city, Khuzestan province, Iran. We followed the clinical outcomes including signs and symptoms, additional diagnostic tests, treatments, and possible complications over six months. The purpose of this study is to aid the better recognition of LMS patients and initiate further interventional research.

Conclusions: Most of the neurological symptoms of LMS recovered or completely resolved after six months. The patient reported an occasional mild headache, reduced left side paresthesia and right side hypoesthesia. Ataxia and fall down on the left side considerably improved. The visual abnormalities were not observed in the eye and neurological examinations, but the patient did report mild dry eyes. Other clinical presentations, such as fine tremors in the right hand, left-sided dysmetria, dizziness, and earache, were completely resolved.
\end{abstract}

Keywords: Ataxia, Emboli, Hiccup, Lateral Medullary Syndromes, Posterior Inferior Cerebellar Artery Syndrome, Stroke, Thrombosis, Vertebral Artery, Vertigo, Wallenberg's Syndrome

\section{Introduction}

Lateral medullary syndrome (LMS), also known as Wallenberg syndrome or posterior inferior cerebellar artery (PICA) syndrome, is a rare cerebrovascular accident, comprising $2.5 \%$ of the ischemic strokes (1). Often, LMS is caused by thrombosis or emboli of the vertebral artery, and less commonly the occlusion of PICA, superior middle, or inferior medullary artery (2). Other causes of this syndrome are hemorrhage, dissection of the vertebral artery, cavernous angioma, and tumors (2). In this syndrome, the posterolateral part of the medulla oblongata in the brain stem is affected (3). This part receives arterial blood supply from PICA, and PICA is a branch of vertebral artery (4).

A wide range of clinical manifestations is found in LMS patients, depending on the involved anatomic structure, including visual abnormalities, decreased pain and temperature sensations on the ipsilateral face and contralateral body, ipsilateral hemiparesis, vertigo, ataxia, nausea and vomiting, a nasal voice, dysphonia, hoarseness, weakness or palatal palsy in the side of the body where the lesion resides, the absence of taste sensation, decreased gag reflex and dysphasia, and slurred speech (5). The visual or eye abnormalities in LMS include different types of nystagmus, gaze palsy, saccadic, ipsipulsion, diplopia, the absence of corneal reflex, and Horner's syndrome (6). LMS is characterized by sensory deficits affecting the trunk and extremities on the opposite side of the infarction and sensory deficits affecting the face and cranial nervous on the same side of the infarction (3).

Dysphagia is a result of nucleus ambiguous involvement (7). Damage to the spinothalamic tract is the reason for the loss of pain and temperature sensation in the opposite side of the body (8). Infarction of the cerebellum or the inferior cerebellar peduncle causes ataxia (9). In LMS, a damage to the spinal trigeminal nucleus causes the absence of pain on the ipsilateral side of the face, as well as the absence of corneal reflex (5). Nystagmus and vertigo result from the involvement of the vestibular nuclei (6). Damage to the hypothalamo-spinal fibers and the sympathetic nervous system gives rise to Horner's syndrome (4). Paliwal et al. (6) reported a 52-year-old male patient with LMS that presented with difficulty in swallowing, nasal re- 
gurgitation of liquids, altered nasal quality of voice, and rightward deviation of eyes and nystagmus. Over four weeks, the ipsipulsion improved spontaneously. Ibrahim et al. reported a 37-year-old male patient with persistent hiccup after lunch. The neurological examination revealed that horizontal nystagmus, bulbar palsy with dysphagia, and nasal phonation of voice. This researcher did not follow the patient to evaluate the recovered or deteriorated neurological deficit (10).

There is no specific treatment for LMS; treatment is symptomatic (9). The prognosis of LMS is dependent on early and exact diagnosis and good management of this syndrome; however, this syndrome is underdiagnosed (9). With optimal diagnosis and treatment, the prognosis for recovery of LMS is favorable (1). Therefore, the cognition and notice of atypical clinical manifestations, diagnostic tests, and treatment approaches of LMS improve the management of this condition. On the other hand, a few case reports have followed the outcomes and disabilities of LMS patients after discharge from the hospital. In this paper, we report the clinical presentation, treatment process, and outcomes of an LMS patient six months after discharge from the hospital, which is a strong point of the present study. The purpose of this study is to aid the better recognition and management of LMS as an unusual type of ischemic stroke. In addition, this report can be a basis for further interventional research on LMS.

\section{Case Presentation}

A 45-year-old man was admitted to the Emergency Room of Imam Mosa Kazem Hospital of Mahshahr city, Khuzestan province, Iran, on 3 October 2016. The patient presented with an acute headache, given a score of 9 out of 10 on a visual analog scale (VAS) of pain, diagnosed with vertigo, persistent hiccup, and left side paresthesia lasting for three days. He was a known case of ischemic heart disease (IHD), hypertension (HTN), and Percutaneous Trans Coronary Angioplasty (PTCA) for the past two years. In addition, he had undergone coronary artery bypass graft (CABG) two years ago. The patient was taking the following medications prior to admission: Amlodipine, Sertraline, Betahistine, Clopidogrel, Atorvastatin, Metoprolol, and Aspirin tablets. The patient had a family history of laryngeal cancer; his sister had died from laryngeal cancer seven years ago, and his father was diabetic, who had died one year ago (Table 1 ).

After admission to the emergency room (ER) and consulting with a neurologist, vital signs were evaluated by the neurologist, including heart rate, respiratory rate, blood pressure, and temperature. Blood pressure was measured

\begin{tabular}{|c|c|}
\hline Item & Value \\
\hline Height, cm & 167 \\
\hline Weight, kg & 85 \\
\hline BMI, $\mathbf{k g} / \mathrm{m}^{2}$ & 30.57 \\
\hline \multicolumn{2}{|l|}{ Vital sign on admission } \\
\hline Heart rate $(\mathrm{HR})$, beat/min & 82 \\
\hline Respiratory rate ( $\mathrm{RR})$, beat/min & 18 \\
\hline Blood pressure (BP), mmHg & $129 / 88$ \\
\hline Temperature, ${ }^{\circ} \mathrm{C}$ & 37.1 \\
\hline \multirow[t]{4}{*}{ Past Medical History (PMH) } & IHD from two past years ago \\
\hline & HTN from two past years ago \\
\hline & PTCA on two years ago \\
\hline & CABG on two years ago \\
\hline \multirow[t]{2}{*}{ Family history } & Father was diabetic. \\
\hline & $\begin{array}{l}\text { His sister suffered from } \\
\text { laryngeal cancer. }\end{array}$ \\
\hline \multicolumn{2}{|l|}{ Laboratory findings (on admission) } \\
\hline Blood Sugar (BS), mg/dL & 131 \\
\hline Blood Urea Nitrogen (BUN), mg/dL & 19 \\
\hline Creatinine $(\mathrm{Cr}), \mathrm{mg} / \mathrm{dL}$ & 0.8 \\
\hline Sodium, meq/L & 137 \\
\hline Potassium, meq/L & 4.5 \\
\hline White Blood Cells (WBC), $\times 1000 / \mu$ & 11.7 \\
\hline Hemoglobin (Hb), mg/dL & 15 \\
\hline Platelets, $\times 1000 / \mu$ & 197 \\
\hline Prothrombin Time (PT), s & 13 \\
\hline International Normalized Ratio(INR) & 1 \\
\hline $\begin{array}{l}\text { Activated Partial Thromboplastin } \\
\text { Time (aPTT), } s\end{array}$ & 25 \\
\hline
\end{tabular}

with a sphygmomanometer (GAMMA, HEINE, made in Germany) that was calibrated before measuring. Then, a physical exam was performed by the neurologist. Head and neck exams revealed the presence of headaches, vertigo, and hiccups; cardiovascular and chest exams of parameters such as heart and lung sounds were to distinguish the clinical signs of respiratory distress; skin exam focused on facial pain, anhidrotic, right or left deviation of lip or other parts of face; eye and ear exam was performed for ptosis, pupil size, and reaction to light, anisocoria, ear discharge, and pain; abdominal and pelvic exam evaluated the signs of gastrointestinal and neurological disorders; and limb and extremities exam revealed paresthesia and history of gaiting ataxia before admission. Neurological exams included mental status exam, level of consciousness and ori- 
entation, cranial and peripheral nervous system exam, sensory and motor exam, including touch, pain, vibration and temperature sensation, muscle strength, muscle tone, and force. Reflexes, such as deep tendon reflex, corneal reflex, and gag reflex, were evaluated. Another neurological exam was a balance exam that included finger to noise and heel to knee tests. A Romberg test was not performed due to the decreased level of consciousness and risk of fall down. The findings of the physical and neurological examinations revealed that the patient was drowsy, oriented to TPP (Time, Place, Person) and co-operative. Heart and lung exams were normal. The patient had a fine tremor in his right hand, face, and right leg hypoesthesia, ataxia and fall down on the left side that had progressed in the last week, visual abnormalities, including partial ptosis on the left eyelid, anisocoria, pupils reactive to light, and left-sided Horner's syndrome. In addition, the finger to nose test revealed dysmetria on the left side of the body. In this research, eye exam and ear exam were performed by ophthalmoscope and otoscope (Keeler model, made in England), respectively. These investigated instruments were calibrated before use in this study. Subsequently, the patient was recommended to have complete bed rest and transferred to the Intensive Care Unit (ICU). The patient was hospitalized for one week in the ICU. During the ICU stay, the patient was conscious and left the bed after two days. He complained of an earache and dizziness when walking after complete bed rest.

\subsection{Investigations}

The laboratory tests were done on admission to ER according to physician order, which included BS, BUN, Cr, Na, K, CBC, PT, PTT, INR, Troponin I, Lactic Dehydrogenase (LDH), Liver Function Test (LFT), Creatine Phosphokinase (CPK), T3, T4, and Thyroid Stimulating Hormone (TSH). Some laboratory tests, including Triglycerides (TG), Cholesterol (Chol), Low-Density Lipoprotein cholesterol (LDL), High-Density Lipoprotein cholesterol (HDL), and urinalysis were done during ICU stay. In addition, some laboratory tests such as FBS, BUN, Cr, Na, K, CPK, LDH, CBC, PT, PTT, and INR were performed daily. Other lab tests were performed as needed, according to physician order. All lab tests were normal. The laboratory tests were done at the laboratory unit of Imam Musa Kazem Hospital, with devices and instruments including Sysmex (KXZIN), Mindray (BC-3000 Plus), ICUBio (imagic-M7), DIRUi CS-300-B, and Caretium. The instruments were calibrated before blood and urine sample analyzing.

On admission, electrocardiography (ECG) Bionet 2000, electroencephalography (EEG) Bionet 2000, chest x-ray, and brain Computed Tomography (CT) scan were performed. The instruments were calibrated before use. The
ECG showed a normal sinus rhythm; EEG and brain CT were normal. The color Doppler sonography of carotid and vertebral artery, Transcranial Doppler Test (TCD), brain magnetic resonance imaging (MRI), and magnetic resonance angiography(MRA) were performed on days 1,2,3, and four after admission to the ICU, respectively. The physician referred the patient to an equipped medical center for Computed Tomography Angiography (CTA) after hospital discharge.

The findings of color Doppler sonography of both sides of the carotid and vertebral arteries revealed the presence of a partially calcified atheromatous plaque measured 7.7 $\times 1.3 \mathrm{~mm}$ seen in the posterior wall of the right carotid bulb, and another similar plaque measured $9.6 \times 1.8 \mathrm{~mm}$ in the posterior wall of the proximal Right Internal Carotid Artery (RICA), which did not cause a significant luminal narrowing (20 - 30\%). In addition, a small partially calcified plaque measuring $9.6 \times 1.9 \mathrm{~mm}$ was seen in the posterior wall of the proximal left Internal Carotid Artery (LICA) without significant luminal narrowing ( 20\%); both sides of the vertebral arteries showed no sign of hypoplasia or atheromatous plaque and normal flow direction without any sign of flow reversal or significant narrowing (Figure 1).

TCD is a diagnostic test for CVA to evaluate blood flow velocity in blood vessels. Figure 2 shows the mean blood flow velocity in brain vessels. The figure reveals the obstruction of brain vessels due to the reduced mean blood flow values, decreased blood flow in Right Vertebral Artery (RVA) (mean flow $=24.6 \mathrm{~cm} / \mathrm{s}$, normal = 38), and mildly increased Intra Cranial Pressure (ICP) according to increasing pulsatility index (PI), which its normal value is 0.5 - 1.9 (Figure 2).

Brain computed tomography showed no acute infarct or hematoma, but brain MRI showed a few flair hyperintense foci in the cerebellar white matter, suggesting that there were small vessel ischemic changes (Figure 3). Brain MRA without contrast revealed no sign of significant stenosis or vascular malformation.

Therefore, the cerebrovascular event was diagnosed as LMS with clinical features, with a pathognomonic finding of LMS in the brain MRI.

Imam Mosa Kazem Hospital is affiliated to the treatment management of Khuzestan social security organization that is a governmental organization. The employed instruments were calibrated before use in this study. Demographic and specific data related to disease were asked from the patient or his family, and other data were extracted from the patient's record or obtained from physical and neurological exams. 

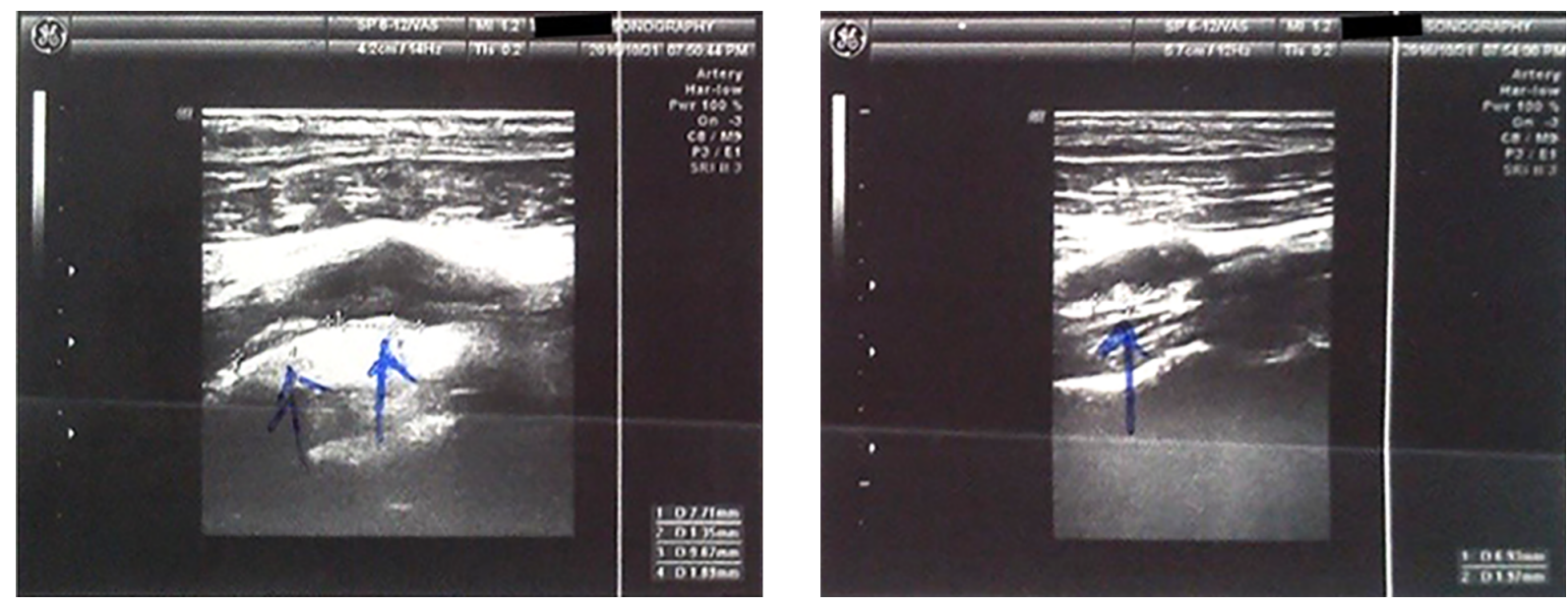

Figure 1. The right and left plaque observed in the internal carotid artery using color Doppler sonography of carotid and vertebral arteries
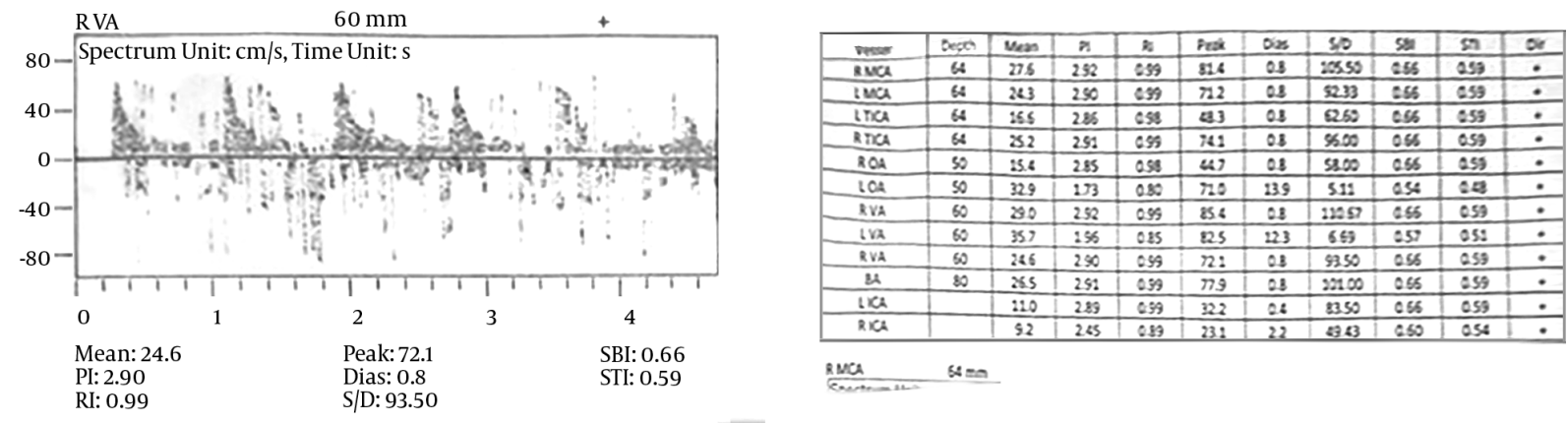

Figure 2. The mild intracranial pressure that was seen rising in the right vertebral artery flow wave using Transcranial Doppler

\subsection{Treatment}

For the management of LMS, antiplatelet drugs (Aspirin, Clopidogrel), an anticoagulant drug (Heparin continual infusion for 24 hours), proton pump inhibitors (Pantoprazole), a $\beta_{1}$ - blocker agent (Metoprolol), and statins (Atorvastatin) were administered to the patient by a neurologist. After three days, the patient's blood pressure increased and antihypertensive drugs (Amlodipine, Losartan) initiated. Clopidogrel and Heparin discontinued and Warfarin started. In addition, Ketorolac, Betahistine, and Novafen were provided to the patient for the treatment of a headache, earache, and dizziness. The administration schedule of medications on admission to ICU was as follows:

1. Tab (tablet) ASA $80 \mathrm{mg}$ Per Oral (PO) daily

2. Tab Clopidogrel $75 \mathrm{mg}$ PO daily

3. Tab Atorvastatin $20 \mathrm{mg}$ PO every night (Qhs)

4. Ampule (Amp) Heparin 5000 Unit Sub Cutaneous (SC) twice a day (BID)
5. Amp Pantoprazole $40 \mathrm{mg}$ Intra Venous (IV) daily

After two days of admission to the ICU, according to clinical manifestations, laboratory tests, and diagnostic investigations, the physician started continuous intravenous heparin $1000 \mathrm{U} / \mathrm{h}$ for $24 \mathrm{~h}$. Three days after admission, the physician discontinued Tab Clopidogrel and continuous intravenous heparin, and initiated Amlodipine 5 mg PO daily, Tab Losartan 25 mg PO three times daily (TID), Tab Warfarin 5 mg PO daily, and Amp Ketorolac $30 \mathrm{mg}$ IV stat injected. After five days in ICU, Tab Novafen $20 \mathrm{mg}$ PO TID, Tab Betahistine 8 mg PO BID, and Tab Nortriptyline 25 mg PO were administered daily.

\subsection{Follow-up}

We followed the clinical outcomes of the patient six months after discharge through telephone and appointments. In addition, the researcher asked a neurologist to visit the patient and exam physical and neurological 

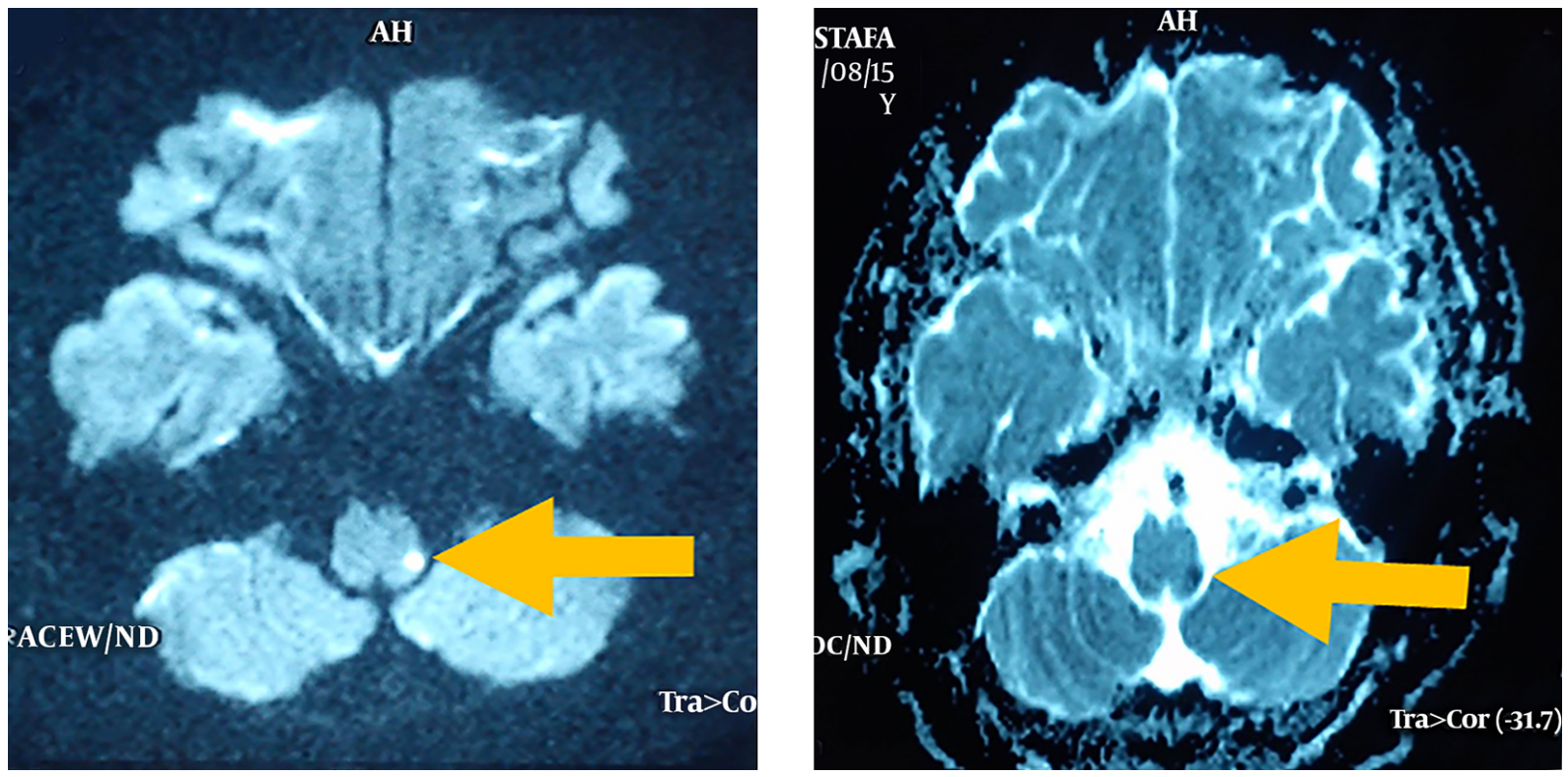

Figure 3. Left medullary lacunar infarction in brain magnetic resonance imaging as indicated by the arrows

sings after six months of discharge. In telephone and appointment conversations, the researcher asked about sign and symptoms, recovered or deteriorated clinical manifestations, possible complications, other diagnostic investigations, and treatment methods of LMS during the six months ago.

Mostly, the patient expressed signs and symptoms of recovery. For example, he reported an occasional mild headache versus an acute headache that had occurred six months ago, and the left side paresthesia and right side hypoesthesia were limited to the right leg and foot sole after six months. Ataxia and fall down on the left side were considerably improved and were limited to higher altitudes. The eye and visual abnormalities were not observed in the eye and neurological examinations, but the patient reported mild dry eyes. Other clinical presentations, such as fine tremors in the right hand, left-sided dysmetria, dizziness, and earache, were completely resolved. Table 2 shows the comparison between the symptoms in our study and other similar research.

\section{Discussion}

LMS is a type of rare ischemic stroke, which is often caused by thrombosis or embolism in the heart or great vessels (9). The severity of clinical manifestations and emergency intervention and monitoring will be dependent on the cause and size of the affected area in the brainstem (10). In severe events of LMS, patients have developed respiratory failure requiring intubation $(11,12,14)$; for example, Tanaka et al. (11) and Sivakumar et al. (14) reported LMS patients with central hypoventilation as a complication of LMS requiring intubation and mechanical ventilation.

In previous studies, different types of visual abnormalities have been discussed, such as Horner's syndrome, diplopia, saccadic, gaze palsy, ipsipulsion, and nystagmus among LMS patients $(5,6,15)$. Tyagi et al. reported $71 \%$ and $54 \%$ of PICA infarcts resulting in ataxia- and gaze-evoked nystagmus, respectively (5). In our case, the patient had mild ptosis on the left eyelid, anisocoria, pupil reactivity to light, and left side Horner's syndrome.

A different severity of dysphagia may be seen in 51-94\% of LMS patients (10). Some LMS patients experience mild dysphagia and recover in 1-2 months after stroke; some experience severe dysphagia and need enteral nutrition for several months or years following the stroke (7). Kim et al. demonstrated dysphagia might be an indicator of poor outcome in acute LMS (16). Nakano et al. reported a 67year-old man with dizziness, dysarthria, and dysphagia as the initial sign and symptoms of LMS. Then, nine days after admission, he presented with asymptomatic hyponatremia and he was diagnosed with syndrome of inappropriate secretion of antidiuretic hormone (SIADH) (13). In our study, the patient had partial dysphagia and could feed orally. The clinical presentations or laboratory data of hyponatremia and SIADH were not seen.

Hiccup is another symptom of LMS that sometimes is 


\begin{tabular}{|c|c|}
\hline Reference & The Symptoms of LMS \\
\hline Tyagi et al. (5) & Vertigo, dysphagia, decreased sensation of the left side and face \\
\hline Saha et al. (9) & $\begin{array}{l}\text { Speech with dysarthric and nasal voice, right side Horner syndrome, loss of pain and temperature sensation in the right side of the face and the } \\
\text { left side of the body }\end{array}$ \\
\hline Ibrahim et al. (10) & Persistent hiccup tolerate to treatment, horizontal nystagmus, dysphagia to fluids \\
\hline Tanaka et al. (11) & A severe headache, central hypoventilation syndrome \\
\hline Raja et al. (12) & Pulmonary edema \\
\hline Nakano et al. (13) & Dizziness, dysarthria, dysphagia, nystagmus, hypothermia, hypoalgesia, ataxia in the left side \\
\hline Current study & Acute headache, vertigo, persisted hiccup, paresthesia in the left leg \\
\hline
\end{tabular}

the initial presentation of LMS (17); Ibrahim et al. described a case report on hiccup in LMS (10). Our patient also had persistent hiccup lasting for three days.

Ataxia and balance impairment are present in almost all patients with LMS and sometimes persist for years even after recovery of other symptoms (17). Ataxia may affect the gait pattern and daily activities of patients with LMS, resulting in a high risk of falling down (18). In our study, the patient suffered from ataxia and fall down on the left side during admission to the hospital. After six months, this symptom restricted to when climbing and hiking mountains.

A strength of this study was good cooperation of the patient for answering to telephone and appointment questions in the ICU and after discharge from the hospital. In addition, the use of reliable, updated references about LMS that is an uncommon CVA was another strength of this paper.

A limitation of this study was the patient that lived in another city of Khuzestan province. However, the good cooperation of the patient and his family for telephone and appointment conversations minimized undesirable effects of this factor.

\subsection{Conclusion}

The outcome of LMS depends on the size and region of the lesion in the brainstem. The signs and symptoms of LMS can be reduced with treatment, but patients' recovery may take weeks or months following treatment. Other patients may be left with significant neurological disabilities for many years. We followed the clinical outcomes of a patient with LMS over six months after discharge. We found that over time, the patient has shown slow, but important progress in all neurological signs and symptoms of LMS.

\section{Footnotes}

Authors' Contribution: Sakine Mazaherpur, Avid Rokni, Ehsan Bastani Far, and Alireza Abdi contributed to concep- tualization, data curation, investigation, project administration, supervision, and writing of the original draft.

Conflict of Interests: No competing interests were disclosed.

Funding/Support: The authors declare that there were no grants involved in supporting this work.

Informed Consent: Written informed consent was obtained from the patient regarding the publication of the patient's details and associated images.

\section{References}

1. Day GS, Swartz RH, Chenkin J, Shamji AI, Frost DW. Lateral medullary syndrome: A diagnostic approach illustrated through case presentation and literature review. CJEM. 2014;16(2):164-70. [PubMed: 24626124].

2. Aravind RS, Athira BM, Mohammed Salim KT. A case report on Wallenberg syndrome. Glob J Add Rehab Med. 2017;4(2). doi: 10.19080/gjarm.2017.04.555634.

3. Nakazato Y, Tamura N, Ikeda K, Yamamoto T. Isolated body lateropulsion caused by lower lateral medullary infarction. eNeurologicalSci. 2017;7:25-6. doi: 10.1016/j.ensci.2017.03.004. [PubMed: 29260021]. [PubMed Central: PMC5721548].

4. Louis DW, Dholakia N, Raymond MJ. Wallenberg syndrome with associated motor weakness in a two-week-postpartum female. Case Rep Neurol.2015;7(3):186-90. doi:10.1159/000440712. [PubMed: 26500545]. [PubMed Central: PMC4611068].

5. Tyagi AK, Ashish G, Lepcha A, Balraj A. Subjective visual vertical and horizontal abnormalities in a patient with lateral medullary syndrome-a case report. Iran J Otorhinolaryngol. 2015;27(78):75-80. [PubMed: 25745615]. [PubMed Central: PMC4344978].

6. Paliwal VK, Kumar S, Gupta DK, Neyaz Z. Ipsipulsion: A forgotten sign of lateral medullary syndrome. Ann Indian Acad Neurol. 2015;18(3):2845. doi: 10.4103/0972-2327.150621. [PubMed: 26425003]. [PubMed Central: PMC4564460].

7. Kim H, Lee HJ, Park JW. Clinical course and outcome in patients with severe dysphagia after lateral medullary syndrome. Ther Adv Neurol Disord. 2018;11. doi: 10.1177/1756286418759864. [PubMed: 29511384]. [PubMed Central: PMC5833167].

8. Katsumata M, Oki K, Nakahara J, Izawa Y, Abe T, Takahashi S, et al. Ipsilateral facial tactile hypesthesia in a patient with lateral medullary syndrome. J Stroke Cerebrovasc Dis. 2015;24(11):e315-7. doi: 10.1016/j.jstrokecerebrovasdis.2015.07.027. [PubMed: 26350694]. 
9. Saha R, Alam S, Hossain MA. Lateral medullary syndrome (Wallenberg's syndrome) - a case report. Faridpur Med Coll J. 2011;5(1). doi: 10.3329/fmcj.v5i1.6813.

10. Ibrahim MH, Fadhil A, Ali SS, Kader SFA, Khalid M, Kumar K, et al. Case report on hiccup and lateral medullary syndrome. Neurosci Med. 2015;6(2):58-61. doi: 10.4236/nm.2015.62010.

11. Tanaka K, Kanamaru H, Morikawa A, Kawaguchi K. Central hypoventilation syndrome complicated with lateral medullary infarction after endovascular treatment of the vertebral artery dissecting aneurysm: A case report. NMC Case Rep J. 2016;3(4):133-6. doi 10.2176/nmccrj.cr.2016-0067. [PubMed: 28664015]. [PubMed Central: PMC5386165].

12. Raja HM, Herwadkar AV, Paroutoglou K, Lilleker JB. Neurogenic pulmonary oedema complicating a lateral medullary infarct. BMJ Case Rep. 2018;2018. doi: 10.1136/bcr-2018-225437. [PubMed: 30054324].

13. Nakano H, Yanase D, Yamada M. Syndrome of inappropriate secretion of antidiuretic hormone (SIADH) associated with lateral medullary syndrome: Case report and literature review. BMC Neurol. 2016;16:119. doi: 10.1186/s12883-016-0641-0. [PubMed: 27461339]. [PubMed Central:
PMC4962364].

14. Sivakumar K, Garcha M, Mehta D, Leary MC, Yacoub HA. Central hypoventilation: A rare complication of Wallenberg syndrome. Case Rep Neurol Med. 2018;2018:1-3. doi: 10.1155/2018/4894820.

15. Kobayashi Z, Numasawa Y, Tomimitsu H, Shintani S. Conjugate eye deviation plus spontaneous nystagmus as a diagnostic sign of lateral medullary infarction. J Neurol Sci. 2016;367:222-3. doi: 10.1016/j.jns.2016.06.017. [PubMed: 27423592].

16. Kim TJ, Nam H, Hong JH, Yeo MJ, Chang JY, Jeong JH, et al. Dysphagia may be an independent marker of poor outcome in acute lateral medullary infarction. J Clin Neurol. 2015;11(4):349-57. doi: 10.3988/jcn.2015.11.4.349. [PubMed: 26256660]. [PubMed Central: PMC4596108].

17. Na EH, Yoon TS, Han SJ. Improvement of quiet standing balance in patients with wallenberg syndrome after rehabilitation. Ann Rehabil Med. 2011;35(6):791-7. doi: 10.5535/arm.2011.35.6.791. [PubMed: 22506207]. [PubMed Central: PMC3309380].

18. McGhie T, Alfred R, Ali A, Gilbert DT. Lateral medullary infarct/Wallenberg syndrome- Jamaica. West Indian Med J. 2012;61(7):1-7. 